\title{
Polarization-sensitive optical coherence tomography-based imaging, parameterization, and quantification of human cartilage degeneration
}

Nicolai Brill

Mathias Wirtz

Dorit Merhof

Markus Tingart

Holger Jahr

Daniel Truhn

Robert Schmitt

Sven Nebelung 


\title{
Polarization-sensitive optical coherence tomography-based imaging, parameterization, and quantification of human cartilage degeneration
}

\author{
Nicolai Brill, ${ }^{a}$ Mathias Wirtz, ${ }^{a}$ Dorit Merhof, ${ }^{b}$ Markus Tingart, ${ }^{c}$ Holger Jahr, ${ }^{c}$ Daniel Truhn, ${ }^{d}$ Robert Schmitt, ${ }^{a, e}$ \\ and Sven Nebelung d,* \\ ${ }^{\text {a}}$ Fraunhofer Institute for Production Technology, Steinbachstraße 17, Aachen 52074, Germany \\ ${ }^{\mathrm{b}}$ RWTH Aachen University, Institute of Imaging and Computer Vision, Kopernikusstraße 16, Aachen 52074, Germany \\ ${ }^{c}$ Aachen University Hospital, Department of Orthopaedic Surgery, Pauwelsstraße 30, Aachen 52074, Germany \\ dAachen University Hospital, Department of Diagnostic and Interventional Radiology, Pauwelsstraße 30, Aachen 52074, Germany \\ ${ }^{\mathrm{e}} \mathrm{RWTH}$ Aachen University, Laboratory for Machine Tools and Production Engineering, Manfred-Weck Haus, Steinbachstraße 19, \\ Aachen 52074, Germany
}

\begin{abstract}
Polarization-sensitive optical coherence tomography (PS-OCT) is a light-based, high-resolution, realtime, noninvasive, and nondestructive imaging modality yielding quasimicroscopic cross-sectional images of cartilage. As yet, comprehensive parameterization and quantification of birefringence and tissue properties have not been performed on human cartilage. PS-OCT and algorithm-based image analysis were used to objectively grade human cartilage degeneration in terms of surface irregularity, tissue homogeneity, signal attenuation, as well as birefringence coefficient and band width, height, depth, and number. Degenerationdependent changes were noted for the former three parameters exclusively, thereby questioning the diagnostic value of PS-OCT in the assessment of human cartilage degeneration. ๑ 2016 Society of Photo-Optical Instrumentation Engineers (SPIE) [DOI: 10.1117/1.JBO.21.7.076013]
\end{abstract}

Keywords: optical diagnostics for medicine; optical coherence tomography; polarization-sensitive optical coherence tomography; cartilage; imaging.

Paper 160282R received Apr. 30, 2016; accepted for publication Jul. 6, 2016; published online Jul. 22, 2016.

\section{Introduction}

Osteoarthritis (OA) is a common clinical pathology that ranks among the top five causes of disability, reaching epidemic proportions in western societies. ${ }^{1}$ With the aging population, absolute patient numbers are expected to continue to rise. ${ }^{2}$ In order to reduce or delay the onset of $\mathrm{OA}$, dedicated methods for timely detection and treatment are critical.

While a multitude of contributory factors have been identified, extracellular matrix (ECM) degeneration is the central feature of OA. ${ }^{3}$ The ECM primarily consists of collagen type-II and proteoglycans and thereby determines the unique compressive and viscoelastic properties of cartilage tissue. The disease process is likely to be initiated by the combination of an unfavorable biomechanical environment and increased cartilage susceptibility. Early disease stages are marked by an upregulation of cellular repair mechanisms to compensate for catabolic and degradation processes ${ }^{4}$ therefore, total collagen content and macroscopic appearance of cartilage tissue remain unaltered. ${ }^{5,6}$ Once these compensatory mechanisms are exhausted, ECM destruction and structural damage progressively develop. In the course of the disease, focal cartilage defects may extend to involve the entire cartilage region, compartment, or joint. ${ }^{7}$

Clinical imaging modalities available today including routinely used radiography, magnetic resonance imaging, and arthroscopy have been demonstrated to lack diagnostic accuracy when the articular surface is still intact, i.e., in early and potentially reversible disease stages. ${ }^{8,9}$ The diagnosis of early OA

${ }^{*}$ Address all correspondence to: Sven Nebelung, E-mail: snebelung@ ukaachen.de requires an imaging modality that sensitively detects and localizes alterations in cartilage structure and composition before the above-mentioned changes turn irreversible.

Optical coherence tomography (OCT) is a light-based, highresolution, real-time, noninvasive, and nondestructive imaging modality that may close this diagnostic gap. Similar to ultrasound, OCT images are generated by the detection of backscattered light characteristics (i.e., echo time delay and intensity). With spatial resolutions similar to low-power histology (i.e., $<10 \mu \mathrm{m}$ ), OCT has been demonstrated to reliably detect cartilage surface and subsurface changes, both in vitro and in vivo as well as in the context of open and arthroscopic joint surgery. ${ }^{9-11}$ Although quantitative parameters were introduced recently in efforts to more objectively and reliably assess cartilage degeneration in conventional OCT (C-OCT),${ }^{12-18}$ the unequivocal differentiation between healthy and early degenerative cartilage remains a challenge to OCT imaging and parameterization.

Polarization-sensitive OCT (PS-OCT) extends the diagnostic performance of conventional OCT by allowing the detection of tissue birefringence. Tissue birefringence is caused by depthdependent differences in collagen fiber organization and orientation ${ }^{19}$ and is of diagnostic value in the study of burn depths in skin $^{20}$ and fiber orientation in intervertebral disks. ${ }^{21}$ Likewise, PS-OCT has been used to study the integrity and orientation of the collagen fiber network in human and animal cartilage. ${ }^{1,22-24}$ A number of studies have suggested an association between characteristic banding patterns and cartilage tissue integrity, ${ }^{10,22,25,26}$ while some recent studies reported

$1083-3668 / 2016 / \$ 25.00$ @ 2016 SPIE 
contrary findings. ${ }^{11,24,27}$ In their pioneering work and using polarized light microscopy (PLM) as the reference imaging technology, Drexler et al. ${ }^{22}$ found cartilage birefringence patterns to be related to collagen organization and its loss to be associated with early cartilage degeneration. These findings were confirmed and built upon by other groups. ${ }^{10,26,28,29}$ In contrast, a number of investigators have recently reported conflicting evidence on cartilage birefringence and its alterations in disease. Xie et al. ${ }^{11}$ demonstrated the differentiation of healthy and degenerative cartilage by PS-OCT to be challenging as either displayed little polarization sensitivity and could not be differentiated by birefringence properties until major matrix alterations at later disease stages, i.e., fibrous tissue formation, were observed. Inline with these findings, several groups have demonstrated both the presence and absence of tissue birefringence in cartilage samples judged by histology to be normal, as well as in samples with early degenerative changes. ${ }^{23,24,27}$

As yet, the clinical relevance of tissue birefringence remains to be fully elucidated. The studies mentioned above have a number of limitations, particularly in the context of clinical requirements. For once, mere qualitative grading of birefringence without quantification of banding characteristics may introduce observer dependence. Up to now, PS-OCT images of human cartilage in various grades of degeneration have not been thoroughly parameterized, quantified, and investigated with respect to their pathological relevance. Likewise, the principal transferability of previous studies to the clinical setting may be limited as animal cartilage has been used primarily, which is marked by distinct differences to human cartilage ${ }^{30}$ and deeper tissue assessment beyond the superficial layer may not be feasible due to technical limitations of the PS-OCT devices used.

This study aimed to parameterize and quantify birefringence properties in PS-OCT images obtained from human cartilage samples with a special focus on the algorithm-based image analysis of birefringence characteristics and their pathological correlation. This study's hypothesis was that these parameters were associated with cartilage degeneration as determined by conventional OCT and histology.

\section{Materials and Methods}

\subsection{Cartilage Sample Preparation}

For this study, a total of four patients (three male, one female; mean age 57.2 years [range 50 to 63 years]) were recruited at the Department of Orthopedic Surgery of the University Hospital Aachen. Both informed consent of individual patients and Institutional Ethical Review Board approval (by the local Ethic Committee for Clinical Trials; AZ EK 157/13) were obtained before the study. Patients underwent total knee replacement due to severe primary OA as diagnosed both clinically and radiographically. Intraoperatively, cartilage-bone samples of various degrees of degeneration were harvested from the central lateral femoral condyles for reasons of topoanatomic consistency. A total of 13 osteochondral samples were thus harvested. Upon harvest, samples were collected in Dulbecco's Modified Eagle Medium (DMEM) (Thermo-Fisher-Scientific, Carlsbad) containing $10 \%$ fetal bovine serum, $100 \mathrm{U} / \mathrm{mL}$ penicillin, $100 \mu \mathrm{g} / \mathrm{mL}$ gentamycin, $1.25 \mathrm{U} / \mathrm{mL}$ amphotericin $\mathrm{B}$, and protease and phosphatase inhibitor cocktail (all from ThermoFisher-Scientific), transferred to the laboratory and kept refrigerated (at $4^{\circ} \mathrm{C}$ ) until use within $24 \mathrm{~h}$. In order to comprehensively assess the PS-OCT procedure, samples of all grades of degeneration with the exception of the bare bone stage were included. Using standard rongeurs, osteochondral samples were cut to standard size $($ width $\times$ length $\times$ depth: $15 \times 15 \times 10 \quad[\mathrm{~mm}])$ with the surface as plane as possible. The midsagittal imaging plane $(0 \mathrm{deg})$ and its orthogonal plane $(90 \mathrm{deg})$ were defined by three notches (ca. $2 \mathrm{~mm}$ each) at the respective sample sides. In order to more comprehensively assess tissue birefringence, samples were scanned at four different orientations $(0,45,90$, and $135 \mathrm{deg}$ ) as referenced to the midsagittal plane (Figs. 1 and 2). Samples were kept hydrated with DMEM + additives as above during preparation. Prior to scanning, samples were cautiously drained on paper to remove excess fluid. Subsequent C-OCT and PS-OCT measurements were performed in air and completed within $2 \mathrm{~min}$.

\subsection{Polarization-Sensitive Optical Coherence Tomography}

Measurements were carried out on a custom-built spectraldomain PS-OCT system using a combination of polarizationmaintaining fibers and free space optics (Fig. 3). The device is based on a super luminescent diode (SLD) with a spectral bandwidth of $100 \mathrm{~nm}$ and centered at $840 \mathrm{~nm}$, allowing image acquisition with a theoretical axial resolution of $3.2 \mu \mathrm{m}$ in air. Optical power incident on the sample was determined as $2.3 \mathrm{~mW}$ at $840 \mathrm{~nm}$ using a suitable photodiode, while the sensitivity of the OCT system was measured as $92.5 \mathrm{~dB}$. A commercially available objective lens (LSM03, Thorlabs, Newton, New Jersey) was used, which allows imaging with a mean spot size of $17 \mu \mathrm{m}$ at the focal plane (defined as the beam diameter, at which the beam power has fallen to $1 / e^{2}$ of its maximum value).

The present PS-OCT system is based on the well-established single-input polarization state (SIPS) setup with circular polarized light on the sample path, which interferes with 45 degpolarized light of the reference path. In principle, the linearly polarized light beam is split into both sample and reference arm inside the probing head of the system. Light being reflected from the sample interferes with the light beam reflected by the reference mirror. A quarter wave plate (QWP) rotated by $45 \mathrm{deg}$ ensures circular polarization of the sample beam to make

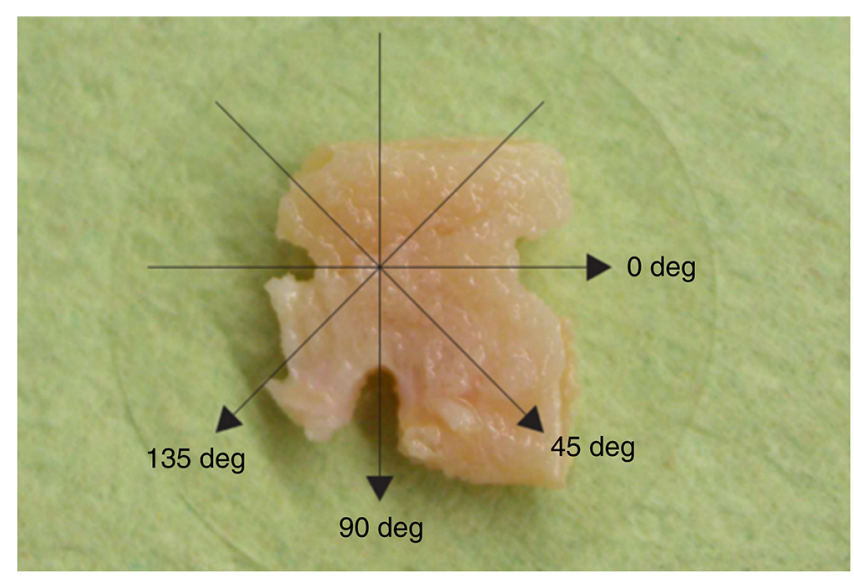

Fig. 1 Definition of the imaging planes as demonstrated by the top view of a moderately degenerated cartilage sample. The midsagittal plane is defined by two notches at opposite sample sides $(0 \mathrm{deg})$. The orthogonal plane $(90 \mathrm{deg})$ is marked by a single notch perpendicular to the midsagittal plane. 
(a)

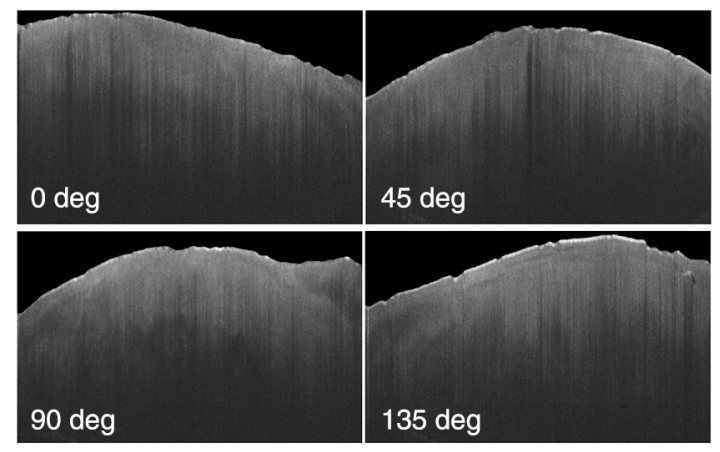

(b)

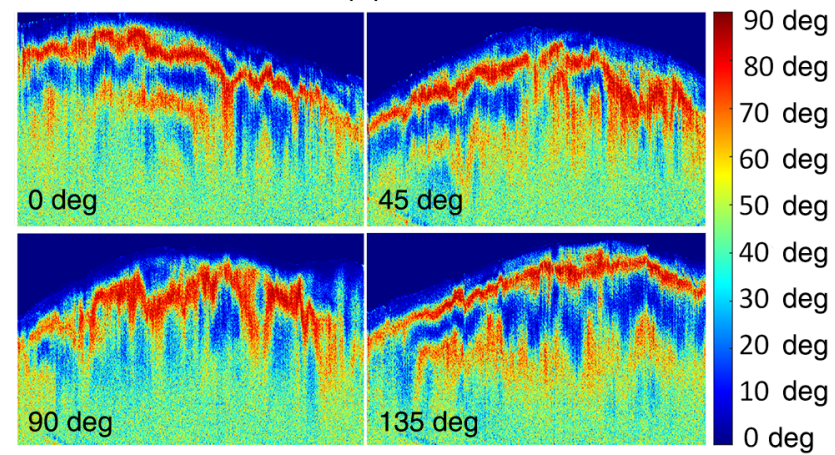

Fig. 2 (a) Conventional intensity OCT images and (b) the corresponding PS-OCT images of the sample presented in Fig. 1 are displayed. Color coding of PS-OCT images: dark blue represents 0 deg phase retardance, whereas red represents $90 \mathrm{deg}$. In addition to the midsagittal plane $(0 \mathrm{deg})$, samples were imaged at three further angles: 45, 90, and 135 deg (see Fig. 1). Total PS-OCT image width corresponds to 8 and $1.5 \mathrm{~mm}$ in depth. For demonstration purposes, noise and autocorrelation artefacts above the top surface are not displayed.

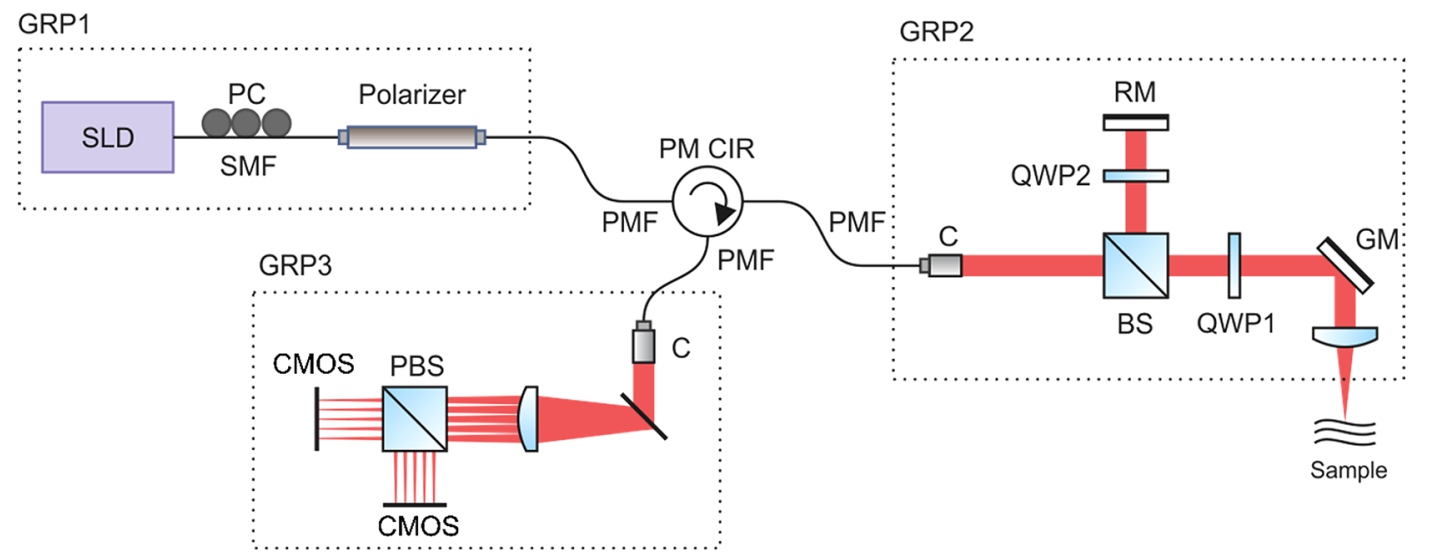

Fig. 3 Diagram of the PS-OCT system used. The system consists of three different groups (GRP). Light generation (GRP1), measurement (GRP2), and detection (GRP3); PC, polarization controller; SMF, single-mode fiber; PMF, polarization-maintaining fiber; PM CIR, polarization-maintaining circulator; C, collimator; BS, beam splitter; QWP1/2, quarter wave plates rotated by $45 / 22.5$ deg, respectively; RM, reference mirror; GM, galvanometer mirror; PBS, polarizing beam splitter; SLD, superluminescent diode; CMOS, complementary metal-oxide-semiconductor.

retardation measurements invariant of sample rotations. ${ }^{31-33}$ The spatial offset between reference mirror and reflecting plane within the sample causes a distinctive modulation of the interference pattern, which allows the reconstruction of sample reflectivity at the respective sample depths. Rather than using two separate spectrometers, the polarization beam splitter has been integrated behind the grating and lens system. The two perpendicular polarization state amplitudes $A_{V}$ and $A_{H}$ are detected separately by two CMOS line scan cameras (Basler sprint spL4096-140k, Basler AG, Germany). The cameras have $2 \times 4096$ pixels each. The vertical binning mode was used so that the number of pixels was set to $1 \times 4096$ with a pixel size of $10 \mu \mathrm{m} \times 20 \mu \mathrm{m}$. The region-of-interest was set to 2048 pixels, yielding an A-scan rate (i.e., axial reflectivity image) of $140 \mathrm{kHz}$. Data processing is performed in real time using a graphical processing unit.

The A-scans of both channels are reconstructed by the application of a fast Fourier transformation (FFT). Sample reflectivity
$R(z)$ and retardation $\eta(z)$ are calculated according to the following equations: ${ }^{31}$

$$
\begin{aligned}
& R(z) \sim A_{V}(z)^{2}+A_{H}(z)^{2}, \\
& \eta=\arctan \left[\frac{A_{V}(z)}{A_{H}(z)}\right]
\end{aligned}
$$

In this study, cross sections (i.e., B-scans) of the samples were obtained by lateral scanning using a galvoscanner setup. B-scans consisted of $1000 \times 1024$ pixels $(8 \times 3.17 \mathrm{~mm})$. For reasons of setup and measurement standardization, cartilage samples were assessed on a linear translation stage with a rotating platform. A polarization-maintaining optical fiber (PMF; length: $10 \mathrm{~m}$ ) was used to connect the measuring head with the PS-OCT base unit. Inline with the method proposed by Al-Qaisi and Akkin, ${ }^{32}$ this setup was chosen as the PMF length 
offsets the typical ghost images caused by cross coupling and shifts these to a less disturbing area of the image.

\subsection{Verification of Phase Retardance}

Phase retardance was verified using a Berek's polarization compensator (New Focus Model 5540). This method has been used before $^{34,35}$ and is based on the retardance variability created by tilting a uniaxial crystalline material. For the measurement process, the Berek's polarization compensator was positioned between the PS-OCT measuring head and a reflective surface. The compensator was then rotated step-wise from 0 to 8 (retardation indicator), corresponding to a phase retardance of 0 to $180 \mathrm{deg}$. After every 0.5 increment, $1000 \mathrm{~A}$-scans were obtained and averaged using the PS-OCT device above. The phase retardance was calculated according to the manufacturer's instructions. Of note, the optic axis was set to 0 deg for all measurements. The measured phase retardance was plotted against the set value of phase retardance (Fig. 4) and overall good agreement between the measured and the expected data points was found. Although the general linear correlation is clearly visible, Fig. 4 also reveals some deviation, primarily at the set phase retardance of 40 to $70 \mathrm{deg}$, which may be due to the circulator and imperfect alignment of the QWP.

\subsection{Image Processing of C-OCT and PS-OCT Data}

As the OCT system is capable of acquiring conventional intensity OCT images as well as retardation images simultaneously, subsequent image parameterization and quantification were based on these images (exemplified by Fig. 2). Structural parameters obtained by the former included surface irregularity [optical irregularity index (OII)], tissue homogeneity [optical homogeneity index $(\mathrm{OHI})$ ], and signal penetration [optical attenuation index (OAI)] as described before. ${ }^{12,14,17,36}$ Briefly, Savitzky-Golay filtering was applied to surface data matrices to obtain smoothed surface topographies. OII as a measure of surface integrity was defined as the standard deviation (SD) between the actual and the smoothed surface topography and calculated in absolute terms. OHI as a measure of tissue homogeneity was determined by analysis of A-scan gradients along the tissue depth and detection of contrast changes. OAI as a measure of signal propagation and imaging depth was

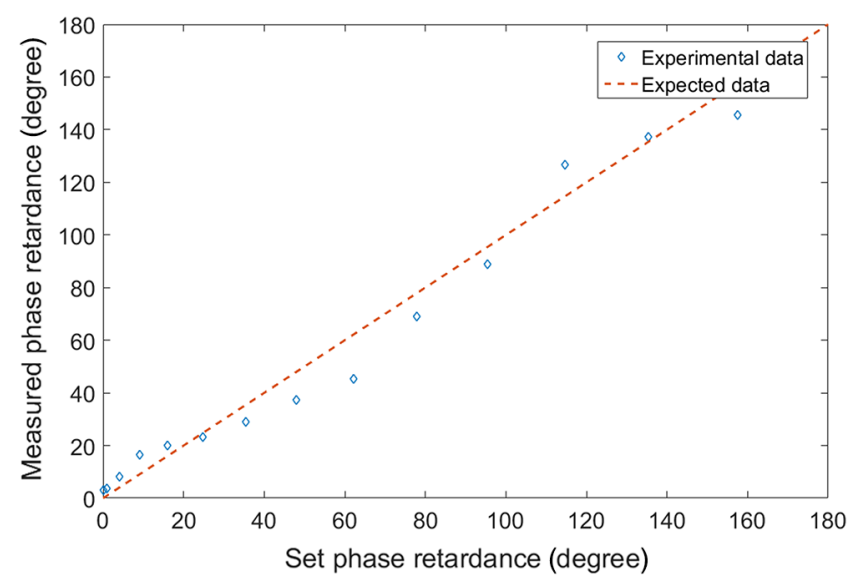

Fig. 4 Verification of the phase retardance. The measured phase retardance is plotted against the set phase retardance (blue circles). Expected values (red-dashed line). determined by detecting the last pixel above the noise level for every A-scan. Of note, OII, OHI, and OAI were determined for the four individual measurement positions per sample (i.e., 0 , 45, 90, and $135 \mathrm{deg}$ ).

PS-OCT image-derived parameters included the birefringence coefficient (BRC), number of detectable bands (NDB), and banding characteristics in retardation images, i.e., depth, height, and width of respective bands. Analysis was performed using custom image processing routines implemented in MATLAB (Mathworks Inc., Natick, Massachusetts).

In addition to ghost artifacts, time-invariant speckle noise is most relevant to subsequent image processing steps due to its potential of severe signal interference. Therefore, additional noise reduction methods were applied prior to processing. Using a modification of the volumetric data acquisition mode, a spatial compounding technique was applied and 50 adjacent B-scans were obtained while laterally deflecting the sample beam over a total lateral distance of $55 \mu \mathrm{m}$. This series of successive B-scans was then averaged to obtain a smoothed B-scan for subsequent analyses ${ }^{37}$ (Fig. 5).

A combination of Canny filtering, ${ }^{38}$ graph theory, and active contours $^{39}$ was used for surface detection in structural OCT images. Image contours were detected by employing canny edge detection, which was used to calculate an adjacency matrix. False edges and ghosting as the result of reflexes and cross coupling in polarization-maintaining fiber-based OCT systems $^{33}$ were addressed by applying graph theory and active contour analysis. For every edge pixel, its nearest neighbor in the forward direction (i.e., the next image column) was detected. A directed graph was derived from this neighborhood and its edges weighted with the distances calculated during the nearest neighbor search. Starting from the left-most node, a path was traversed in the direction of the nearest neighbor. Thus, outliers were never reached during the traverse and could be excluded. Gaps produced by removing the outliers were closed by piecewise cubic interpolation as described by Fritsch and Carlson. ${ }^{40}$ In cartilage surfaces with clefts, where the nearest neighbor approach might cause jumps, the detected surface was used as a starting condition for an active contour refinement of the topology. ${ }^{39}$

Retardation images underwent correction in terms of unwrapping due to the computation of the phase difference $\eta$ between both polarization states $\left(A_{V}, A_{H}\right)$ via arctangent. Retardation images were smoothed by application of combined wavelet thresholding and Savitzky-Golay filtering (Fig. 6).

Phase retardance information at respective minima and maxima (i.e., A-scan depths at exceeding multiples of $\pi / 2$ ) were detected on the smoothed images. BRC was defined as phase retardation versus imaging depth and calculated by averaging the cumulative retardation of each A-scan at its maximum imaging depth; here, cumulative means the sum of the unwrapped retardation, while the maximum imaging depth is the same, which is used for determining OAI.

As banding characteristics in retardation images have not yet been objectively quantified in cartilage samples, PS-OCT-based algorithms, which are capable of automatic quantification of banding characteristics in terms of depth, width, and height, were developed: by definition, retardation maxima and minima represented the center of their respective bands. A $k$-nearest neighbor search was performed to derive a context between neighboring extrema. By applying a threshold $T_{c}$ to the graph edges, nodes with weak connectivity (i.e., high distance) 

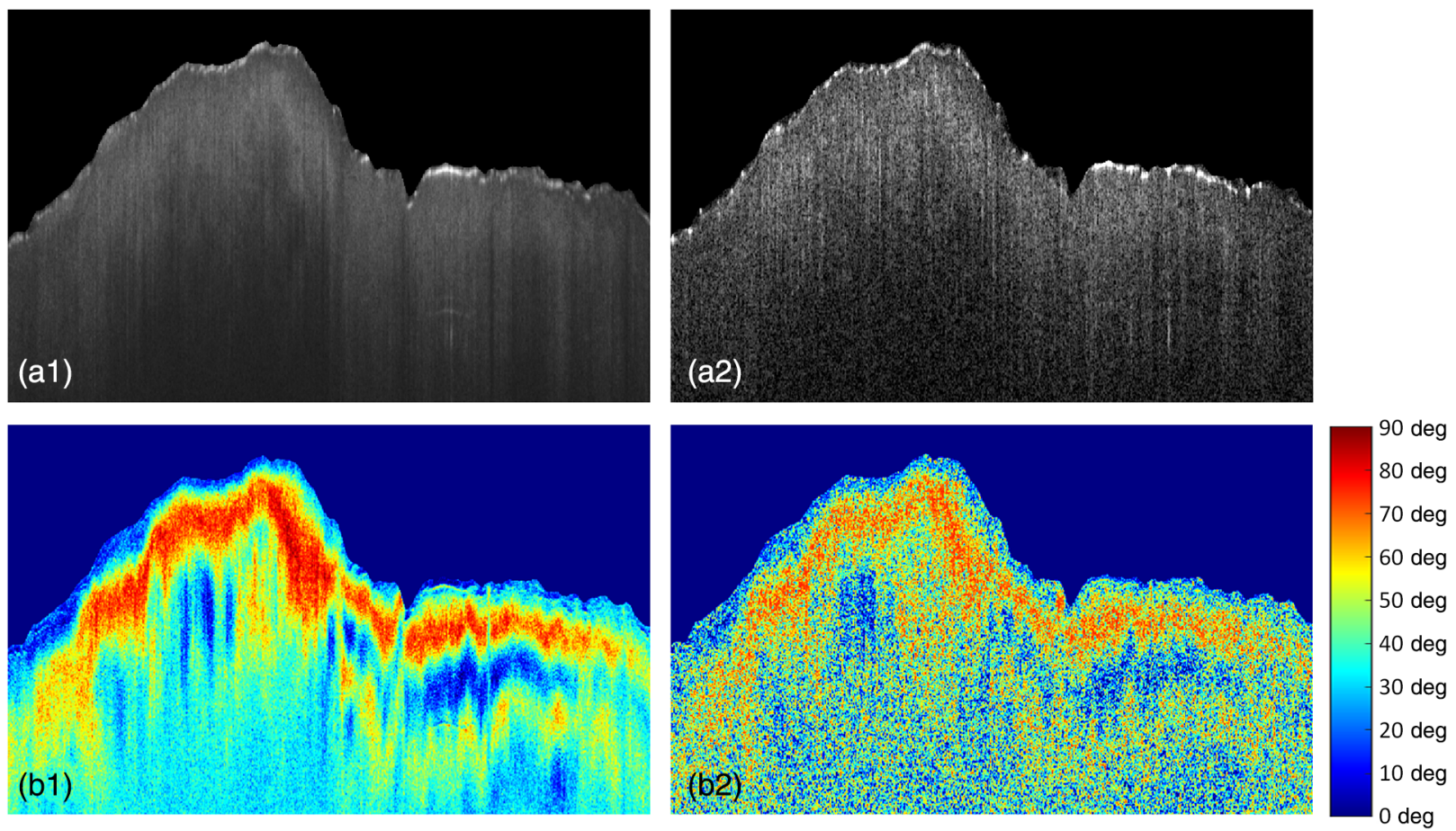

Fig. 5 A spatial compounding technique was used to obtain smoothed B-scans. To this end, (a1) and (b1) 50 adjacent B-scans over a lateral distance of $55 \mu \mathrm{m}$ were individually detected and averaged; (a2) and (b2) the original B-scan is displayed for illustration. (a) Intensity-based images and (b) PS-OCT images with the color-coding as defined in Fig. 2. Total images' width corresponds to 8 and $1.5 \mathrm{~mm}$ in depth.

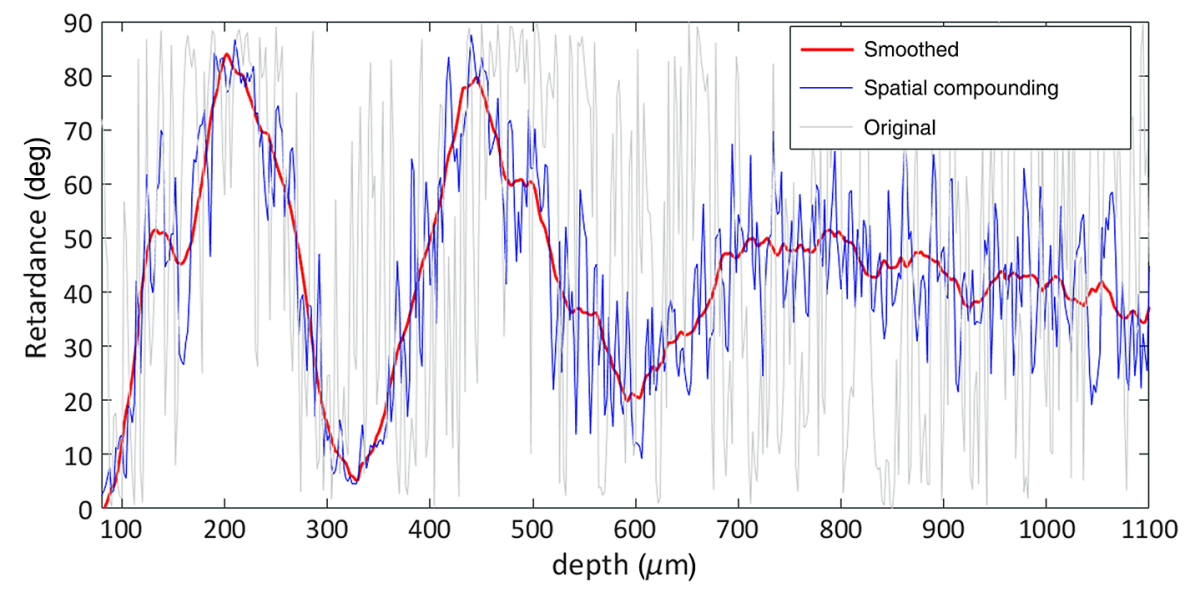

Fig. 6 Smoothing process for an exemplary A-scan of the tissue sample displayed in Fig. 5. First, a spatial compounding technique was used for noise reduction of the original A-scan (gray line) to obtain the averaged A-scan (blue line), which was then filtered by a combination of wavelet thresholding and Savitzky-Golay filtering to obtain a smoothed signal (red line) for further analysis.

were separated to leave only strongly connected clusters behind. Based on the previous works by Kasagarod et al., ${ }^{41}$ who demonstrated maxima or minima to be separated by a depth-wise distance of at least 200 to $300 \mu \mathrm{m}$ in each A-scan, $T_{c}$ was set to 50 pixels $(125 \mu \mathrm{m})$. Stable results were coherently demonstrated even in tissues that exhibited strong and dense birefringence patterns (Fig. 7).

Connected bands found by this approach were then quantified by calculating their relative lateral extent as compared to the detected surface (width, $w_{\mathrm{B}}$ ), their average distance from the tissue surface over the sample width (depth, $d_{\mathrm{B}}$ ), and their band height (mean height, $h_{\mathrm{B}}$; maximum height, $h_{\mathrm{B}, \max }$; minimum height, $h_{\mathrm{B}, \mathrm{min}}$ ) over the sample width as displayed in Fig. 8. Boundaries between two neighboring bands were defined as those depths where retardation values passed the halfway mark toward the next maximum or minimum (i.e., average of adjacent local maximum and minimum), respectively.

\subsection{Histological Analysis}

After OCT measurements, samples underwent decalcification and fixation in Ossa fixona (Diagonal, Muenster, Germany), 


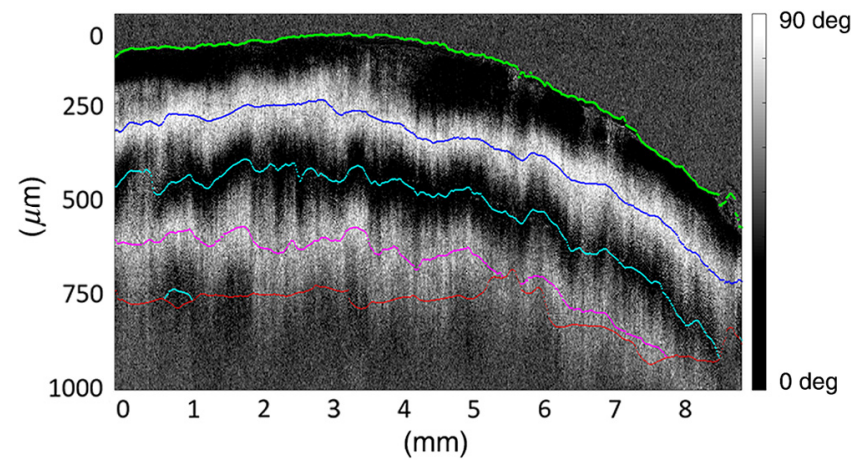

Fig. 7 Retardation image of an intact cartilage tissue sample exhibiting strong banding. Detected phase maxima are marked magenta and blue; phase minima are displayed as cyan. The first connected band was selected and printed in blue. Green and red lines mark the tissue surface and imaging depth, respectively.

sectioning along the midsagittal plane and embedding in paraffin. Hence, two sample halves were obtained, cut to $5 \mu \mathrm{m}$ sections and stained with hematoxylin and eosin (HE), safranin $\mathrm{O}$ (Saf-O), and picrosirius red according to standard protocols. ${ }^{42}$ Images of stained slices were taken using either conventional light microscopy (CLM; Leica, Wetzlar, Germany) or PLM (Zeiss, Jena, Germany). PLM images were captured with two polarized filters set perpendicular to each other and assessed in terms of collagen fiber organization, orientation, and integrity.

Blinded qualitative histological and OCT-based grading of cartilage degeneration was performed by two experienced investigators according to a modified version of the degenerative joint disease (DJD) ${ }^{43}$ classification (HE staining): DJD 0, normal cartilage; DJD 1, surface irregularities (wrinkling, fraying, laminar separations); DJD 2, clefts limited to the superficial zone; DJD $3 / 4 / 5$, clefts extending to the transitional/deep/calcified zone; DJD 6, complete tissue disorganization, fibrous tissue replacement; and DJD 7, complete cartilage erosion. Likewise, qualitative cartilage features as assessed by CLM and PLM were assessed using the cartilage matrix grading scale as suggested by David-Vaudey (David-Vaudey Score, DVS ${ }^{42}$ ) (Table 1; HE and Picrosirius Red staining).

For subsequent analyses, samples were grouped according to their histological appearance: no degeneration (nd, i.e., DJD 0),

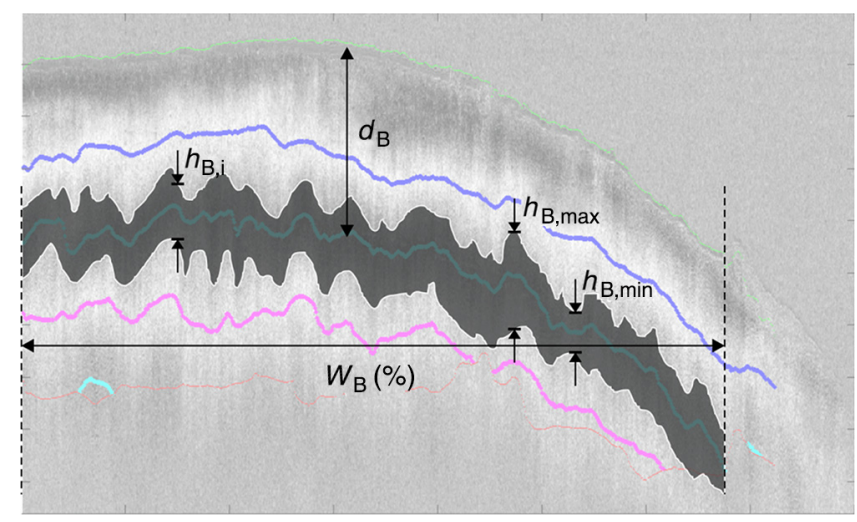

Fig. 8 Banding characteristics as parameterized and quantified by the retardation banding algorithm. $w_{\mathrm{B}}$, band width as compared to the detected surface; $d_{\mathrm{B}}$, mean band depth; $h_{\mathrm{B}, \min }, h_{\mathrm{B}, \max }$, minimum and maximum band height; $h_{\mathrm{B}}$, average band height. Same sample image as in Fig. 7.
Table 1 CLM and PLM classification of cartilage matrix characteristics as described by David-Vaudey et al. ${ }^{42}$

\begin{tabular}{lll} 
Grade & \multicolumn{1}{c}{ CLM characteristics } & \multicolumn{1}{c}{ PLM characteristics } \\
\hline 0 & No surface irregularities & $\begin{array}{l}\text { Presence of birefringence } \\
\text { in surface and deep zone }\end{array}$ \\
1 & Mild surface irregularities & $\begin{array}{l}\text { Disruption of birefringence } \\
\text { in surface }\end{array}$ \\
2 & $\begin{array}{l}\text { Significant surface } \\
\text { fibrillation }\end{array}$ & $\begin{array}{l}\text { Disruption of birefringence } \\
\text { in superficial zone }\end{array}$ \\
3 & $\begin{array}{l}\text { Significant surface and } \\
\text { moderate transitional zone } \\
\text { degeneration } \\
\text { Significant degeneration } \\
\text { extending into radial zone }\end{array}$ & $\begin{array}{l}\text { Breakdown of birefringence } \\
\text { in superficial zone }\end{array}$ \\
& $\begin{array}{l}\text { Breakdown of birefringence } \\
\text { in superficial and deep } \\
\text { zone }\end{array}$ \\
\hline
\end{tabular}

early degeneration (ed, i.e., DJD 1 and 2), and moderate degeneration (md, i.e., DJD 3 to 5).

\subsection{Statistical Analysis}

Graphpad Prism Software (Version 5.0, GraphPad Software Inc.) was used for statistical analyses. Differences between degeneration groups (nd, ed, and md) were evaluated using one-way ANOVA followed by Tukey's posthoctest. Correlations were evaluated using Pearson's correlation coefficients. Numerical data are expressed as mean values $(M) \pm S D$, while $p$-values $\leq 0.05$ were considered statistically significant. More specifically, significance levels are indicated by [***, highly significant] for $p$-values of $p \leq 0.001$, by [**, significant] for $p$-values of $0.001<p \leq 0.01$ and by [*, barely significant] for $p$-values of $0.01<p \leq 0.05$.

\section{Results}

Histological evaluation revealed DJD grade 0 in 5 samples (nd group), DJD grades 1 and 2 in 0 and 5 samples, respectively (ed group), and DJD grades 3, 4, and 5 in 0,1 , and 2 samples, respectively (md group). Therefore, the nd and ed groups comprised five samples each, whereas the md group comprised three samples. Representative histological images are displayed in Fig. 9.

Table 2 shows outcomes for all parameters. Significant degeneration-dependent differences were found in all C-OCT parameters (i.e., OII, OHI, and OAI) and the polarization microscopy score (i.e., DVS). Of note, group-wise assessment revealed that only OII and DVS allowed significant differentiation of nd and ed cartilage, whereas all of the above-mentioned parameters allowed significant differentiation of nd and md cartilage. None of the polarization-sensitive OCT parameters, i.e., BRC or NDB, was significantly associated with degenerative grades.

Wherever present, the first and second bands of each sample were detected and analyzed in terms of depth, width, and height (Table 3 ). The first and second bands were not significantly different in their banding characteristics with the exception of the first band depth, which was significantly closer to the surface in nd than in ed samples. 


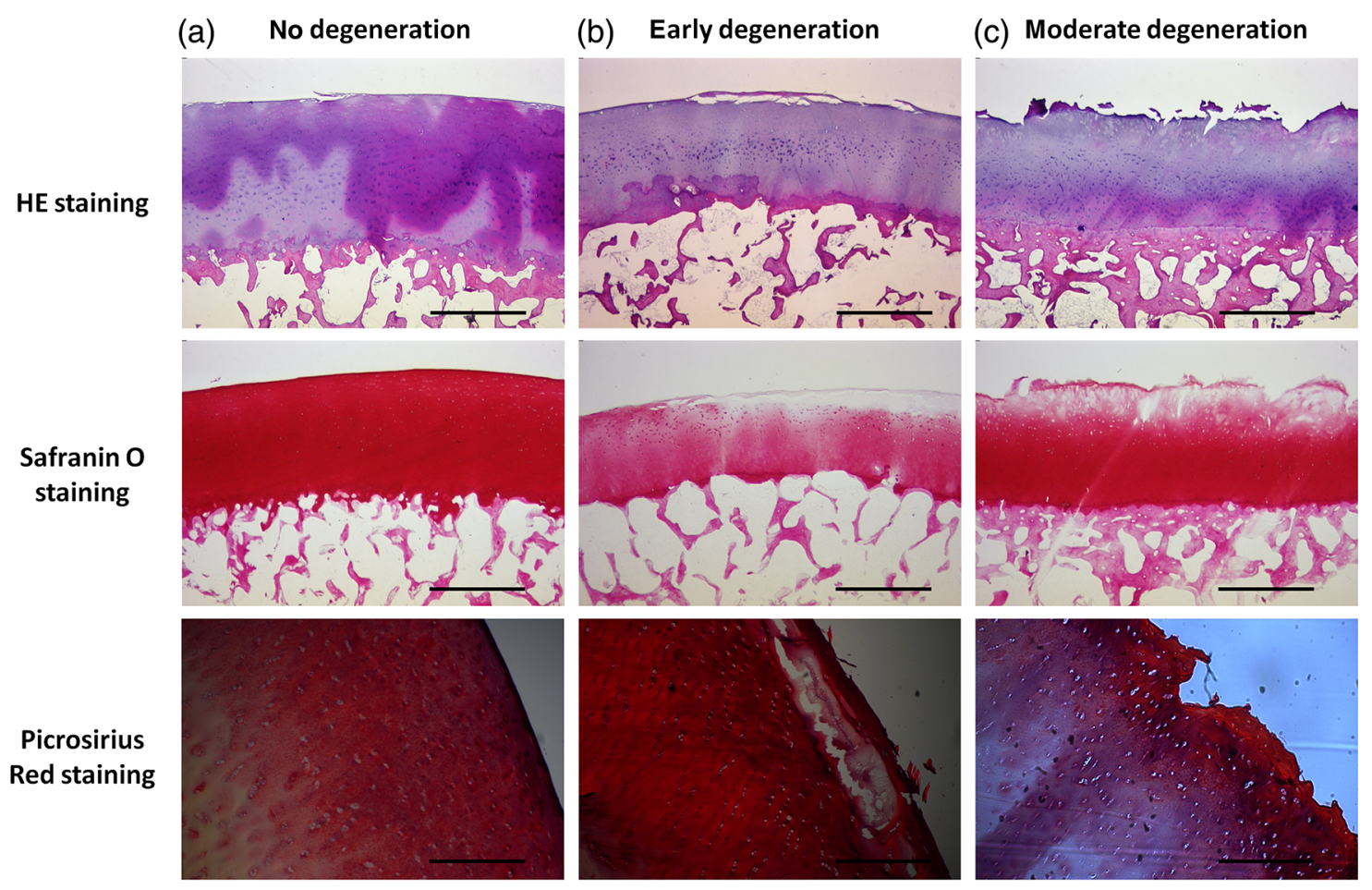

Fig. 9 Representative histological images of samples graded (a) DJD grade 0, (b) grade 2, and (c) grade 4. Scale bars represent $1000 \mu \mathrm{m}$ (HE and Saf-O staining) and $200 \mu \mathrm{m}$ (Picrosirius red staining), respectively.

Throughout all samples, only partially coherent banding was observed, regardless of the degenerative grade.

Inline with the quantitative analysis, qualitative assessment revealed banding characteristics to be unrelated to the degenerative grade (Fig. 10).
Statistically significant correlations were found between a number of PS-OCT, C-OCT, banding, and histological parameters (Tables 4): PS-OCT parameters (i.e., BRC, NDB) were significantly correlated with the first- and second band widths and the second band depth (BRC only). Moreover, a number of

Table 2 Assessment of human cartilage by PS-OCT (BRC, NDB), quantitative (OII, OHI, OAI), and qualitative (DJD) C-OCT parameters as well as conventional and polarized light microscopy parameters.

\begin{tabular}{|c|c|c|c|c|c|c|c|c|c|}
\hline & & $\mathrm{BRC}\left(\times 10^{-4}\right)$ & NDB $(n)$ & Oll (abs) & $\mathrm{OHI}$ (abs) & OAI (abs) & CLM (DJD) & qual. OCT (DJD) & PLM (DVS) \\
\hline \multirow[t]{2}{*}{ nd } & M & 5.3 & 2.8 & 12.7 & 0.6 & 639.6 & 0.0 & 0.4 & 0.2 \\
\hline & SD & 2.2 & 1.0 & 5.3 & 0.3 & 92.6 & 0.0 & 0.9 & 0.5 \\
\hline \multirow[t]{2}{*}{ ed } & M & 3.8 & 2.3 & 34.3 & 0.9 & 609.6 & 1.2 & 1.6 & 2.6 \\
\hline & SD & 0.9 & 0.6 & 13.6 & 0.5 & 109.3 & 0.4 & 0.9 & 1.1 \\
\hline \multirow[t]{2}{*}{$\mathrm{md}$} & M & 234.7 & 1.6 & 65.9 & 2.2 & 418.1 & 4.3 & 4.7 & 3.3 \\
\hline & SD & 1.3 & 0.7 & 11.9 & 1.0 & 82.6 & 1.5 & 0.6 & 0.6 \\
\hline$p$-value & & 0.077 & 0.164 & $<0.001$ & 0.010 & 0.027 & $<0.001$ & $<0.001$ & 0.002 \\
\hline \multirow[t]{3}{*}{ Posthoc testing } & nd versus ed & ns & ns & * & ns & ns & ns & ns & ** \\
\hline & nd versus md & ns & ns & *** & ** & * & *** & *** & ** \\
\hline & ed versus md & ns & ns & $\star \star$ & * & ns & 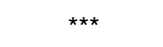 & $\star \star$ & ns \\
\hline
\end{tabular}

Significant differences are bold.

Abbreviations: M, mean; $\pm \mathrm{SD}$, standard deviation; nd, no degeneration; ed, early degeneration; md, moderate degeneration; BRC, birefringence coefficient; NDB, number of detected bands; OII, optical irregularity index; OHI, optical homogeneity index; OAI, optical attenuation index; CLM, conventional light microscopy; DJD, degenerative joint disease classification of cartilage degeneration; PLM, polarized light microscopy; DVS, David-Vaudey score; ns, not significant. 
Table 3 Characteristic parameters of the first and second detectable bands in human cartilage of different degenerative grades as assessed by PS-OCT.

Depth $(\mu \mathrm{m}) \quad$ Width $(\%) \quad$ Mean height $(\mu \mathrm{m})$

\begin{tabular}{|c|c|c|c|c|}
\hline \multicolumn{5}{|c|}{ First band } \\
\hline \multirow[t]{2}{*}{ nd } & M & 255.3 & 62.1 & 255.0 \\
\hline & SD & 23.6 & 29.8 & 26.8 \\
\hline \multirow[t]{2}{*}{ ed } & M & 387.4 & 50.2 & 263.2 \\
\hline & SD & 106.5 & 3.6 & 90.2 \\
\hline \multirow[t]{2}{*}{ md } & M & 243.0 & 27.7 & 158.5 \\
\hline & SD & 47.9 & 15.9 & 66.1 \\
\hline$p$-value & & 0.024 & 0.149 & 0.118 \\
\hline \multirow[t]{3}{*}{ Tukey's } & nd versus ed & * & ns & ns \\
\hline & nd versus md & ns & ns & ns \\
\hline & ed versus md & ns & ns & ns \\
\hline \multicolumn{5}{|c|}{ Second band } \\
\hline \multirow[t]{2}{*}{ nd } & M & 426.8 & 40.3 & 285.0 \\
\hline & SD & 184.9 & 16.3 & 49.1 \\
\hline \multirow[t]{2}{*}{ ed } & M & 389.2 & 24.0 & 272.6 \\
\hline & SD & 72.0 & 8.5 & 127.7 \\
\hline \multirow[t]{2}{*}{ md } & M & 287.5 & 19.2 & 164.4 \\
\hline & SD & 53.0 & 11.6 & 135.3 \\
\hline$p$-value & & 0.488 & 0.109 & 0.382 \\
\hline
\end{tabular}

Depth is the average distance from the tissue surface over the sample width, which is the relative lateral extent as compared to the detected surface, while mean height represents the mean band height over the detected sample width. Abbreviations are given in Table 2.

significant correlations were found for both qualitative and quantitative C-OCT parameters, while their overall correlations to banding parameters were weaker (Table 4).

\section{Discussion}

The most important finding of this study is that PS-OCT imaging parameterization and quantification are feasible and allow for more comprehensive, objective, standardized, and reliable assessment of human cartilage degeneration as compared to C-OCT alone. However, the majority of individual PS-OCT parameters such as birefringence or banding characteristics seem to be unrelated to the samples' degree of degeneration. This is in line with some of the more recent literature data available on the optical properties of cartilage in relation to degeneration, ${ }^{11}$ while other studies have described contrary findings. ${ }^{26,28}$ Xie et al. investigated phase retardation and its depth dependency in relation to cartilage degeneration in bovine samples. They found characteristic mild polarization shifts in normal or moderately diseased hyaline cartilage, whereas multiple strong optical phase retardation shifts were described in severe degeneration marked by fibrocartilaginous replacement. ${ }^{11}$ In contrast, a number of nonhuman ex-vivo studies suggested that OCT birefringence patterns were associated with collagen microstructure integrity, and therefore, hypothesized its presence or absence to be associated with cartilage health or degeneration, respectively. Using single-channel OCT devices without quantification of optical phase retardation, numerous investigators found changes in banding patterns, which were theorized to be the result of osteoarthritic changes..$^{10,22,25}$

As outlined above, our study failed to demonstrate the usefulness of PS-OCT-based imaging and associated algorithms to be beneficial in the grading of cartilage degeneration. Nevertheless, the PS-OCT parameters showed degenerationrelated tendencies: for once, the BRC, i.e., the slope of the phase retardation versus depth, tended to decrease with increasing degeneration. This is inline with the previous data as phase retardation plots obtained in bovine cartilage suggested no considerable difference between the different degeneration grades, although the exact BRCs were not reported. ${ }^{23}$ Investigating healthy and mildly degenerative porcine cartilage, Shyu et al. ${ }^{44}$ described lower BRCs in healthy cartilage and a high degree of variability in mildly degenerative cartilage. These observations could not be confirmed by our study, possibly because of differences in experimental setup, sample characteristics, image analysis, or parameter definition and extraction.

Likewise, banding characteristics were investigated in an effort to further parameterize and quantify cartilage banding. The first and second bands were not significantly different in their banding characteristics with the exception of the first band depth, which was found to be significantly closer to the surface in healthy than in early degenerative cartilage. In view of the fact that (1) the level of significance was weak, (2) this observation could not to be confirmed by the depth of the second band, and (3) otherwise, banding characteristics were not different, this observation should be put into perspective.

By trend, lower numbers of detectable bands were found with increasing degeneration. This may be due to the progressive tissue loss and cartilage thinning that is associated with OA. ${ }^{45}$ Inline with this finding, the depths and mean heights of the first and second band tended to be smallest in moderately degenerative cartilage, i.e., these bands, if detectable, were closer to the cartilage surface. Possible explanations involve the tissue quality, substance, and organization, which is steadily lost in the course of degeneration. Therefore, hyaline cartilage undergoing ECM loss, condensation, erosion, and fibrous replacement may produce bands that are fewer, smaller, and less clearly distinguishable.

Another important aspect of banding is the width of coherent bands. In our study, the first band tended to be wider in terms of coherent banding than the second band. Moreover, band width tended to decrease with degeneration. However, only partially coherent banding was observed throughout all samples, regardless of the degree of degeneration. Despite considerable scientific attention, banding patterns and their pathological significance in the cartilage tissue are still controversial. While a number of studies have made the association between characteristic banding patterns and cartilage tissue integrity, ${ }^{10,22,25,26}$ a number of recent studies reported contrary findings. ${ }^{11,24,27}$ Using PLM as the reference imaging technology, Drexler et al. ${ }^{22}$ found cartilage birefringence patterns to be related to collagen 

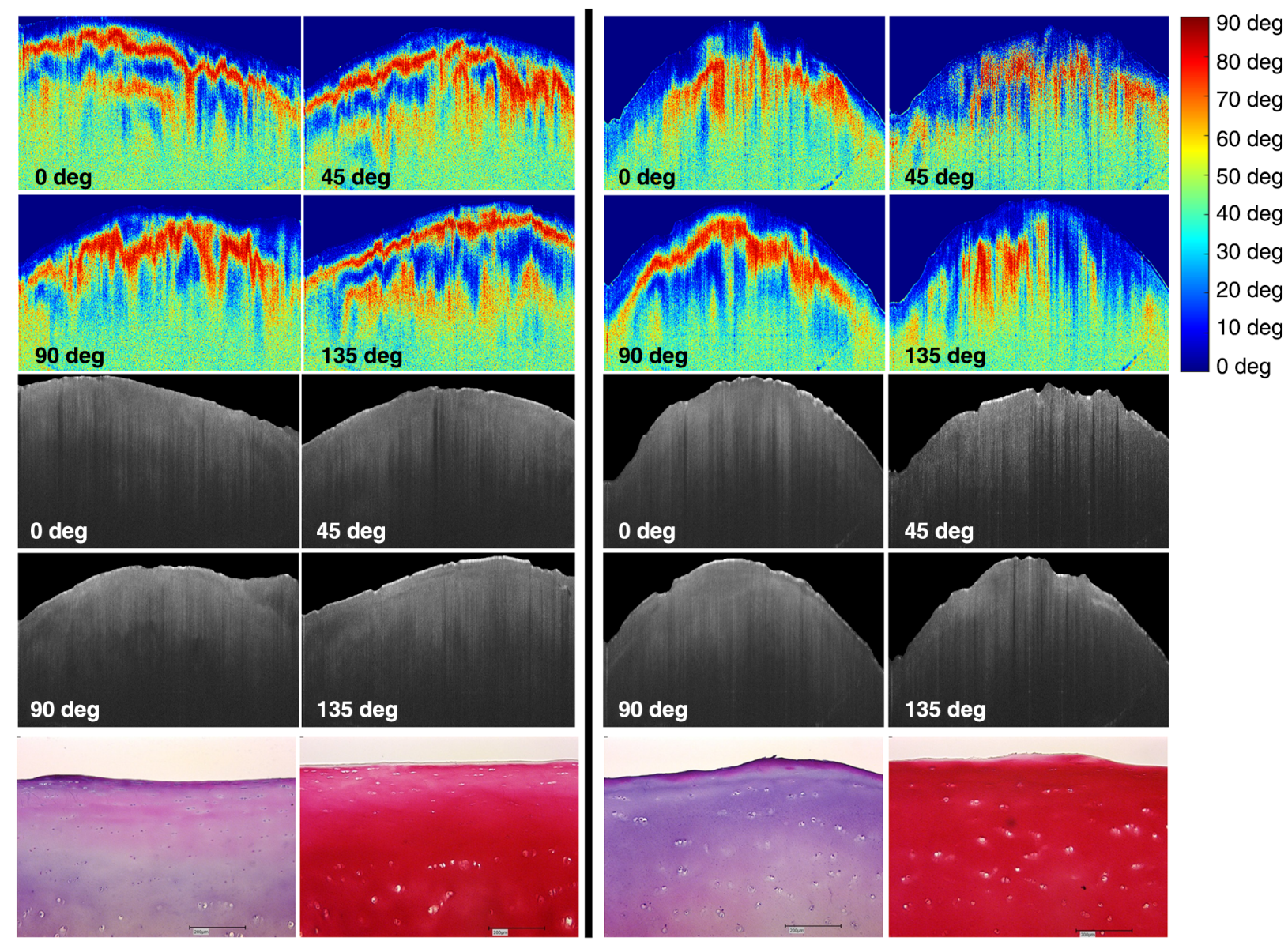

Fig. 10 Retardation images and corresponding histological images of two different samples histologically classified as DJD 0 . Sample 1 exhibited strong banding at all measured angles (left images), whereas sample 2 exhibited only partial and incoherent banding (right images). PS-OCT images (upper images) and conventional intensity OCT images (middle images) are displayed as well as the corresponding histological images (lower images). Here, HE (left) and Saf-O staining (right) of the sample sections indicated that the absence of histological degeneration and scale bars represent $200 \mu \mathrm{m}$. See Figs. 1 and 2 for OCT image details.

organization and its loss to be associated with early cartilage degeneration. This group defined banding of more than $50 \%$ image width as normal, while less or no bands was considered pathological. Likewise, other groups used the presence or absence of tissue birefringence patterns to determine the degree of degeneration in cartilage samples. ${ }^{10,26,28,29}$ In a study on human cartilage tissue assessed ex vivo, the absence of detectable PS-OCT banding patterns was hypothesized to be associated with early OA. ${ }^{29}$ Moreover, the same group assessed early human cartilage degeneration in patients with degenerative meniscal tears. ${ }^{26}$ In these studies, healthy articular cartilage was associated with strong birefringence that was found to be decreased with degeneration. Abnormality was noted if cartilage tissue displayed partial or no banding.

In contrast to the findings above, a number of investigators have recently reported differing findings on cartilage birefringence and its alterations as a function of cartilage status. Xie et al. ${ }^{11}$ demonstrated that PS-OCT may not be used to differentiate healthy from degenerative cartilage as either displayed little polarization sensitivity and was not to be differentiated by birefringence properties until major matrix alterations at later disease stages (i.e., fibrous tissue formation) were observed. Several groups have demonstrated both the presence and absence of polarization sensitivity in cartilage samples judged by histology to be normal as well as in samples with early degenerative changes. ${ }^{23,24,27}$ For once, healthy cartilage showed no optical phase retardation with the optical axis normal to the cartilage surface, i.e., with the incident angle of light at $90 \mathrm{deg}$, while significant polarization sensitivity was present if the incident angle of light was placed off-normal. ${ }^{23}$ Healthy equine cartilage failed to display birefringence banding with the incident angle of light beam normal to the tissue surface, while off-normal (i.e., more horizontal) light angles produced coherent banding. ${ }^{24}$ Also in a study on healthy cartilage samples, Xie et al. ${ }^{23}$ demonstrated differences in banding patterns with the incident angle of light directed at different angles to the surface. With an average curvature of $4.4 \mathrm{~m}^{-1}$ (range, $-20.0 \mathrm{~m}^{-1}$ to $27.2 \mathrm{~m}^{-1}$ ) determined for human femoral condyles, knee cartilage anatomy and geometry are likely to severely affect banding if strict perpendicularity is maintained during measurements. Moreover, healthy cartilage displayed significant variations in polarization sensitivity at different locations in bovine and equine articular cartilage samples. ${ }^{24,27}$

In addition to the overall health status of cartilage, a number of biological and physical factors have been demonstrated to affect optical phase retardation and subsequent banding 
Table 4 Correlations between different qualitative and quantitative conventional and polarization-sensitive OCT and histology parameters.

\begin{tabular}{|c|c|c|c|c|c|c|c|c|}
\hline & & \multicolumn{2}{|c|}{ PS-OCT } & \multicolumn{3}{|c|}{ C-OCT } & \multicolumn{2}{|c|}{ Qualitative } \\
\hline & & $\mathrm{BRC}\left(\times 10^{-4}\right)$ & $\operatorname{NDB}(n)$ & Oll (abs) & $\mathrm{OHI}(\mathrm{abs})$ & OAI (abs) & OCT (DJD) & CLM (DJD) \\
\hline \multirow[t]{2}{*}{ PS-OCT } & $\mathrm{BRC}\left(\times 10^{-4}\right)$ & - & - & - & - & - & - & - \\
\hline & NDB $(n)$ & $0.813 ;<0.001$ & - & - & - & - & - & - \\
\hline \multirow[t]{3}{*}{ C-OCT } & Oll (abs) & $-0.592 ; 0.033$ & $-0.699 ; 0.008$ & - & - & - & - & - \\
\hline & $\mathrm{OHI}$ (abs) & $-0.495 ; 0.085$ & $-0.475 ; 0.101$ & $0.711 ; 0.006$ & - & - & - & - \\
\hline & OAI (abs) & $0.718 ; 0.006$ & $0.759 ; 0.003$ & $-0.718 ; 0.006$ & $-0.604 ; 0.029$ & - & - & - \\
\hline \multirow[t]{2}{*}{ Qualitative } & OCT (DJD) & $-0.536 ; 0.059$ & $-0.657 ; 0.015$ & $0.961 ;<0.001$ & $0.709 ; 0.007$ & $-0.702 ; 0.007$ & - & - \\
\hline & CLM (DJD) & $-0.6668 ; 0.0128$ & $-0.551 ; 0.051$ & $0.796 ; 0.001$ & $0.594 ; 0.032$ & $-0.704 ; 0.007$ & $0.818 ; 0.001$ & - \\
\hline \multirow[t]{3}{*}{ First band } & Depth $(\mu \mathrm{m})$ & $-0.225 ; 0.460$ & $-0.186 ; 0.543$ & $0.086 ; 0.781$ & $-0.234 ; 0.442$ & $0.319 ; 0.288$ & $-0.057 ; 0.853$ & $-0.078 ; 0.800$ \\
\hline & Width (\%) & $0.924 ;<0.001$ & $0.657 ; 0.015$ & $-0.483 ; 0.094$ & $-0.462 ; 0.112$ & $0.733 ; 0.004$ & $-0.425 ; 0.148$ & $-0.629 ; 0.021$ \\
\hline & Mean height $(\mu \mathrm{m})$ & $0.307 ; 0.308$ & $0.471 ; 0.104$ & $-0.604 ; 0.029$ & $-0.410 ; 0.163$ & $0.570 ; 0.042$ & $-0.614 ; 0.025$ & $-0.595 ; 0.032$ \\
\hline \multirow[t]{3}{*}{ Second band } & Depth $(\mu \mathrm{m})$ & $0.715 ; 0.009$ & $0.408 ; 0.188$ & $-0.228 ; 0.475$ & $-0.315 ; 0.319$ & $0.674 ; 0.016$ & $-0.181 ; 0.574$ & $-0.408 ; 0.187$ \\
\hline & Width (\%) & $0.846 ;<0.001$ & $0.714 ; 0.009$ & $-0.630 ; 0.028$ & $-0.576 ; 0.050$ & $0.637 ; 0.026$ & $-0.507 ; 0.093$ & $-0.406 ; 0.190$ \\
\hline & Mean height $(\mu \mathrm{m})$ & $0.416 ; 0.178$ & $0.485 ; 0.110$ & $-0.541 ; 0.069$ & $0.077 ; 0.812$ & $0.577 ; 0.049$ & $-0.520 ; 0.083$ & $-0.636 ; 0.026$ \\
\hline
\end{tabular}

Data are presented as: Pearson's correlation coefficient; $p$-value. Significant correlations are bold. Abbreviations are given in Table 2.

characteristics in PS-OCT images. These include sample dimensions, orientation, topography, localization within the joint, composition, collagen matrix properties, i.e., thickness, orientation, organization, and alignment, and the incident angle of light. ${ }^{11,20,23,24,27}$

Hence, considerable misinterpretation of the collagen organization and/or cartilage health status is possible as optical phase retardation and subsequent banding characteristics are related to the factors above, which are not well controlled as yet. Although C-OCT parameters such as OII, OHI, and OAI were significantly different between degeneration grades, they did not allow for the differentiation of healthy versus early degenerative cartilage. This finding is supported by earlier findings indicating that detection of earliest structural alterations by C-OCT remains elusive, ${ }^{15-17,46,47}$ although the highly sensitive detection of larger-scale structural alterations such as roughness, surface delamination, or cleft formation by C-OCT is undisputed. , $^{9,10,23,48}$

Limitations of our study involve biological and technical aspects. One principle limitation involves the overall limited sample size of 13 human articular cartilage samples harvested from four patients. Due to its exploratory character and technical focus, our study was aimed at assessing the principal feasibility and clinical potential of parameterizing and quantifying PSOCT-derived image characteristics. In consideration of this aspect, future studies need to be performed with greater sample sizes, increased statistical power, and, quite possibly, the inclusion of alternative sample sources such as organ donor networks to obtain samples representative of the entire spectrum of health and disease. The assessment of PS-OCT-derived parameters in the comprehensive evaluation of cartilage degeneration remains to be performed in future studies.
Technical limitations in terms of wavelength and axial depth resolution challenge the comprehensive assessment of collagen microstructure components as an additional determinant of cartilage degeneration. Although histology was defined as the reference standard, additional determination of mechanical and biochemical cartilage properties may allow for more comprehensive assessment of PS-OCT imaging reliability and validity. Future study designs may better control the multitude of factors affecting birefringence as outlined above. In particular, the complex interrelatedness of these factors with the measurement outcome needs to be taken into account to render PS-OCT imaging clinically beneficial in cartilage assessment.

In conclusion, this study demonstrates that PS-OCT imagebased analysis, parameterization, and quantification of human knee cartilage in health and disease are feasible. However, degeneration-dependent differences in PS-OCT derived parameters tended to be minor and their clinical relevance is yet to be fully elucidated. Nevertheless, our study provides an experimental framework for future studies that are aimed at comprehensively and objectively detecting alterations in tissue birefringence as a sign of disease. Structural OCT parameters such as OII, OHI, and OAI may be of diagnostic value in tissues that do not demonstrate disease-related changes in tissue birefringence.

\section{Acknowledgments}

The authors would like to thank Ms. Sophie Lecouturier for her overall technical assistance. Also, the authors do not have any financial, consulting, or personal relationships with other people or organizations to disclose. This research project was supported by the START Program of the Faculty of Medicine, RWTH 
Aachen, Germany, granted to SN (AZ 09/14 [696600] and 120/ 15 [691551]).

\section{References}

1. R. C. Lawrence et al., "Estimates of the prevalence of arthritis and selected musculoskeletal disorders in the United States," Arthritis Rheum 41(5), 778-799 (1998).

2. D. T. Felson, "An update on the pathogenesis and epidemiology of osteoarthritis," Radiol. Clin. North Am. 42(1), 1-9 (2004).

3. D. T. Felson and T. Neogi, "Osteoarthritis: is it a disease of cartilage or of bone?," Arthritis Rheum. 50(2), 341-344 (2004).

4. L. J. Sandell and T. Aigner, "Articular cartilage and changes in arthritis. An introduction: cell biology of osteoarthritis," Arthritis Res. 3(2), 107-113 (2001).

5. A. Changoor et al., "Structural characteristics of the collagen network in human normal, degraded and repair articular cartilages observed in polarized light and scanning electron microscopies," Osteoarthritis Cartilage 19(12), 1458-1468 (2011).

6. T. Franz et al., "In situ compressive stiffness, biochemical composition, and structural integrity of articular cartilage of the human knee joint," Osteoarthritis Cartilage 9(6), 582-592 (2001).

7. G. R. Squires et al., "The pathobiology of focal lesion development in aging human articular cartilage and molecular matrix changes characteristic of osteoarthritis," Arthritis Rheum. 48(5), 1261-1270 (2003).

8. A. J. Palmer et al., "Non-invasive imaging of cartilage in early osteoarthritis," Bone Joint J. 95-B(6), 738-746 (2013).

9. C. R. Chu et al., "Arthroscopic microscopy of articular cartilage using optical coherence tomography," Am. J. Sports Med. 32(3), 699-709 (2004).

10. X. Li et al., "High-resolution optical coherence tomographic imaging of osteoarthritic cartilage during open knee surgery," Arthritis Res. Ther. 7(2), R318-323 (2005).

11. T. Xie et al., "Determination of characteristics of degenerative joint disease using optical coherence tomography and polarization sensitive optical coherence tomography," Lasers Surg. Med. 38(9), 852-865 (2006).

12. S. Nebelung et al., "Quantitative OCT and MRI biomarkers for the differentiation of cartilage degeneration," Skeletal Radiol. 45, 505-516 (2016).

13. T. Viren et al., "Comparison of ultrasound and optical coherence tomography techniques for evaluation of integrity of spontaneously repaired horse cartilage," J. Med. Eng. Technol. 36(3), 185-192 (2012).

14. S. Nebelung et al., "Three-dimensional imaging and analysis of human cartilage degeneration using optical coherence tomography," J. Orthop. Res. 33(5), 651-659 (2015).

15. N. Brill et al., "3D Human cartilage surface characterization by optical coherence tomography," Phys. Med. Biol. 60(19), 7747-7762 (2015).

16. N. Brill et al., "Optical coherence tomography-based parameterization and quantification of articular cartilage surface integrity," Biomed. Opt. Express 6(7), 2398-2411 (2015).

17. S. Nebelung et al., "Morphometric grading of osteoarthritis by optical coherence tomography-an ex vivo study," J. Orthop. Res. 32(10), 13811388 (2014).

18. S. Saarakkala et al., "Quantification of the optical surface reflection and surface roughness of articular cartilage using optical coherence tomography," Phys. Med. Biol. 54(22), 6837-6852 (2009).

19. J. P. Arokoski et al., "Decreased birefringence of the superficial zone collagen network in the canine knee (stifle) articular cartilage after long distance running training, detected by quantitative polarised light microscopy," Ann. Rheum. Dis. 55(4), 253-264 (1996).

20. S. M. Srinivas et al., "Determination of burn depth by polarizationsensitive optical coherence tomography," J. Biomed. Opt. 9(1), 207-212 (2004).

21. S. J. Matcher, C. P. Winlove, and S. V. Gangnus, "The collagen structure of bovine intervertebral disc studied using polarization-sensitive optical coherence tomography," Phys. Med. Biol. 49(7), 1295-1306 (2004).

22. W. Drexler et al., "Correlation of collagen organization with polarization sensitive imaging of in vitro cartilage: implications for osteoarthritis," J. Rheumatol. 28(6), 1311-1318 (2001).
23. T. Xie et al., "Use of polarization-sensitive optical coherence tomography to determine the directional polarization sensitivity of articular cartilage and meniscus," J. Biomed. Opt. 11(6), 064001 (2006).

24. N. Ugryumova et al., "The collagen structure of equine articular cartilage, characterized using polarization-sensitive optical coherence tomography," J. Phys. D 38, 2612-2619 (2005).

25. J. M. Herrmann et al., "High resolution imaging of normal and osteoarthritic cartilage with optical coherence tomography," J. Rheumatol. 26(3), 627-635 (1999).

26. C. R. Chu et al., "Clinical optical coherence tomography of early articular cartilage degeneration in patients with degenerative meniscal tears," Arthritis Rheum. 62(5), 1412-1420 (2010).

27. T. Xie et al., "Topographical variations in the polarization sensitivity of articular cartilage as determined by polarization-sensitive optical coherence tomography and polarized light microscopy," J. Biomed. Opt. 13(5), 054034 (2008).

28. D. M. Bear et al., "Optical coherence tomography grading correlates with MRI T2 mapping and extracellular matrix content," J. Orthop. Res. 28(4), 546-552 (2009).

29. C. R. Chu et al., "Clinical diagnosis of potentially treatable early articular cartilage degeneration using optical coherence tomography," J. Biomed. Opt. 12(5), 051703 (2007).

30. K. A. Athanasiou et al., "Interspecies comparisons of in situ intrinsic mechanical properties of distal femoral cartilage," J. Orthop. Res. 9(3), 330-340 (1991).

31. E. Gotzinger, M. Pircher, and C. K. Hitzenberger, "High speed spectral domain polarization sensitive optical coherence tomography of the human retina," Opt. Express 13(25), 10217 (2005).

32. M. K. Al-Qaisi and T. Akkin, "Polarization-sensitive optical coherence tomography based on polarization-maintaining fibers and frequency multiplexing," Opt. Express 16(17), 13032 (2008).

33. E. Gotzinger et al., "Polarization maintaining fiber based ultra-high resolution spectral domain polarization sensitive optical coherence tomography," Opt. Express 17(25), 22704 (2009).

34. Z. Ding et al., "Quantitative single-mode fiber based PS-OCT with single input polarization state using Mueller matrix," Biomed. Opt. Express 6(5), 1828-1843 (2015).

35. C. Hitzenberger et al., "Measurement and imaging of birefringence and optic axis orientation by phase resolved polarization sensitive optical coherence tomography," Opt Express 9(13), 780-790 (2001).

36. F. de Bont et al., "Evaluation of single-impact-induced cartilage degeneration by optical coherence tomography," BioMed Res. Int. 2015, 486794 (2015).

37. D. L. Marks, T. S. Ralston, and S. A. Boppart, "Data analysis and signal postprocessing for optical coherence tomography," in Optical Coherence Tomography: Technology and Applications, W. Drexler and J. G. Fujimoto, Eds., pp. 405-424, Springer (2008).

38. J. Canny, "A computational approach to edge detection," IEEE Trans. Pattern Anal. Mach. Intell. PAMI-8(6), 679-698 (1986).

39. T. F. Chan and L. A. Vese, "Active contours without edges," IEEE Trans. Image Process. 10(2), 266-277 (2001).

40. F. N. Fritsch and R. E. Carlson, "Monotone piecewise cubic interpolation,” SIAM J. Numer. Anal. 17(2), 238-246 (1980).

41. D. K. Kasaragod et al., "Experimental validation of an extended Jones matrix calculus model to study the 3D structural orientation of the collagen fibers in articular cartilage using polarization-sensitive optical coherence tomography," Biomed. Opt. Express 3(3), 378-387 (2012).

42. E. David-Vaudey et al., "T2 relaxation time measurements in osteoarthritis," Magn. Reson. Imaging 22(5), 673-682 (2004).

43. S. Nebelung et al., "Morphometric grading of osteoarthritis by optical coherence tomography—an ex vivo study," J. Orthop. Res. 32, 1381-1388 (2014).

44. J.-J. Shyu et al., "Diagnosis of articular cartilage damage by polarization sensitive optical coherence tomography and the extracted optical properties," Prog. Electromagnet. Res. 91, 365-376 (2009).

45. R. U. Kleemann et al., "Altered cartilage mechanics and histology in knee osteoarthritis: relation to clinical assessment (ICRS Grade)," Osteoarthritis Cartilage 13(11), 958-963 (2005).

46. H. Jahr, N. Brill, and S. Nebelung, "Detecting early stage osteoarthritis by optical coherence tomography?," in Biomarkers: Biochemical Indicators of Exposure, Response, and Susceptibility to Chemicals, pp. 1-7 (2016). 
47. S. Nebelung et al., "Towards optical coherence tomography-based elastographic evaluation of human cartilage," J. Mech. Behav. Biomed. Mater. 56, 106-119 (2015).

48. Y. Pan et al., "Hand-held arthroscopic optical coherence tomography for in vivo high-resolution imaging of articular cartilage," J. Biomed. Opt. 8(4), 648-654 (2003).

Nicolai Brill studied physics and received his master's degree from the RWTH Aachen University, Germany. He has been working as a scientist in the Production Metrology Department, Fraunhofer IPT since 2010 concentrating on the field of optical coherence tomography as well as fiberoptic microprobes for interferometric distance metrology and the development of customized measurement systems for industrial applications. Since 2015, he has been an entrepreneur in the field of optical technologies.

Mathias Wirtz studied electrical engineering at the RWTH Aachen University, Germany, where he received his diploma in 2014. During his diploma thesis at the Production Metrology Department, Fraunhofer IPT, Aachen, Germany, he got scientifically involved in various aspects of OCT-based imaging, in particular of orthopedic soft tissues. Since 2015, he has been working in the field of software engineering.

Dorit Merhof has been the head of the Institute of Imaging and Computer Vision, RWTH Aachen University, Germany, since 2013. After completing her studies in computer science and her PhD thesis on medical image analysis and visualization at the University of Erlangen-Nuremberg, Germany, she worked at Oxford (UK) and Konstanz (Germany) before coming to Aachen.

Markus Tingart has been the head physician of the Department of Orthopaedic Surgery, University Hospital Aachen, Germany, since October 2010. After studying medicine at the RWTH Aachen University, he received his orthopedic and traumatology training in
Cologne and Regensburg. As a scientist and clinician, Prof. Tingart is a member of several professional bodies, has been awarded a number of prestigious grants and fellowships, and has lectured extensively on all aspects of orthopedics.

Holger Jahr is the current head of the research laboratory of the Department of Orthopaedic Surgery at the University Hospital Aachen. After studying biology and completing his PhD thesis, he joined the Departments of Internal Medicine and Orthopaedics, Erasmus MC University Medical Center, Rotterdam, The Netherlands, before coming to Aachen.

Daniel Truhn studied physics and medicine at the RWTH Aachen University, Germany. He is currently a resident in the Department of Diagnostic and Interventional Radiology at the University Hospital Aachen. He earned his doctorate degree in medicine in 2013 with a thesis on the shielding characteristics of MR-compatible PET detectors. His main research interests cover musculoskeletal imaging techniques including MRI and OCT.

Robert Schmitt has been a professor at the Laboratory for Machine Tools and Production Engineering (WZL) at RWTH Aachen University, since July 2004. He is a member of the boards of directors of Fraunhofer IPT and WZL. Following his studies in electrical engineering, he worked as a chief engineer at WZL. In 1997, he joined the quality-management executive staff at a leading producer of commercial vehicles.

Sven Nebelung completed his medical education with honors at the RWTH Aachen University and completed his basic surgical training at the Department of Orthopedic Surgery, University Hospital of Aachen, Germany. After that, he joined the Department of Diagnostic and Interventional Radiology at the same institution. His areas of clinical and scientific interest are innovative imaging modalities around cartilage and meniscus as well as sports medicine. 